\title{
Teste diagnóstico de resiliência potencial \\ O radar da resiliência organizacional
}

\author{
por Teresa Rodrigues, António Silva Ribeiro, Lia Vasconcelos e Benoît Robert
}

RESUMO: Neste artigo, elaborado a partir de um estudo de caso realizado entre 2011 e 2015, explora-se o conceito de resiliência potencial na Marinha portuguesa durante o choque orçamental ocorrido em Portugal (2011). O trabalho teórico e empírico orientado para o desenvolvimento de um instrumento de diagnóstico de resiliência potencial por captação de ecos foi concebido para mapear ecos de resiliência potencial, designados por ecos de protorresiliência. Os resultados da aplicação indicam que o Radar-R possui um conjunto de benefícios não intrusivos que permitem aos atores do sistema estruturarem o pensamento de forma clara e focada, num ambiente ambíguo, de modo a identificarem alavancas potenciadoras para a organização responder estrategicamente à mudança. Pretende-se contribuir para estimular o desenvolvimento de trabalhos teóricos e empíricos de resiliência das organizações nas comunidades científicas e organizacionais/empresariais de língua portuguesa. Palavras-chave: Resiliência; Avaliação; Mapeamento; Organizações; Estratégia

Teresa Rodrigues

te.rodrigues@gmail.com

Doutoranda em Ciências do Ambiente, Universidade Nova de Lisboa, Faculdade de Ciências e Tecnologia, Campus da Caparica, 2829-516 Caparica, Portugal. Investigadora em resiliência das organizações, formadora de professores e Coach.

Estudiante de doctorado en Ciencias Ambientales, Universidad Nueva de Lisboa, Facultad de Ciencias y Tecnología, Campus de Caparica, 2829-516 Caparica, Portugal. Investigadora en resiliencia organizacional, formadora de profesores y Coach.

$\mathrm{PhD}$ candidate in Environment Science, Nova University of Lisbon, Faculty of Sciences and Technology, Caparica Campus, 2829-516 Caparica, Portugal. Researcher in organizational resilience, trainer and Coach.

\section{António Silva Ribeiro}

antonio.silva.ribeiro@marinha.pt

Doutorado em Ciência Política, Instituto superior de Ciências Sociais e Políticas. Professor Catedrático Convidado, Instituto Superior de Ciências Sociais e Políticas, 1300-663 Lisboa, Portugal. Exerce atualmente o cargo de Chefe do Estado-Maior da Armada e Autoridade Marítima Nacional (CEMA-AMN), é um académico especializado nas áreas de Estratégia, Ciência Política e História.

Doctorado en Ciencias Políticas, Instituto de Ciencias Sociales y Políticas. Profesor Catedrático invitado, Instituto Superior de Ciencias Sociales y Políticas, 1300-663 Lisboa, Portugal. Ejerce actualmente el cargo de Jefe de Estado Mayor de la Armada y de la Autoridad Marítima Nacional (CEMA-AMN), académico especializado en las áreas de Estrategia, Ciencias Políticas e Historia.

$\mathrm{PhD}$ in Political Science, Institute of Social and Political Sciences. Invited Full Professor, Institute of Social and Political Sciences, 1300-663 Lisbon, Portugal. Currently holds the Position Chief of Portuguese Naval Staff and National Maritime Authority (CEMA-AMN), is a specialized academic areas Strategy, Political Science and History. 


\section{Prueba de diagnóstico del potencial de resiliencia}

\section{El radar de la resiliencia organizacional}

RESUMEN: En este artículo, elaborado a partir de un estudio de caso realizado entre 2011 y 2015, se explora el concepto del potencial de resiliencia en la Marina portuguesa durante la crisis financiera ocurrida en Portugal (2011). El trabajo teórico y empírico está orientado al desarrollo de un instrumento de diagnóstico del potencial de resiliencia por captación de ecos diseñados para mapear ecos de potencial de resiliencia, designados como ecos de proto-resiliencia. Los resultados indican que el Radar- $\mathrm{R}$ tiene un conjunto de beneficios no intrusivos que permiten a los actores del sistema estructurar el pensamiento de forma clara y enfocada, en un ambiente ambiguo, a fin de identificar palancas que potencien responder a la organización de forma estratégica al cambio. Se pretende contribuir a estimular el desarrollo de trabajos teóricos y empíricos de resiliencia en las organizaciones de las comunidades científicas y organizacionales/empresariales de lengua portuguesa.

Palabras clave: Resiliencia; Evaluación; Mapeo; Organizaciones; Estrategia

\section{Lia Vasconcelos}

Itv@fct.unl.pt

Doutorada em Engenharia do Ambiente/Sistemas Sociais,

Faculdade de Ciências e Tecnologia, Universidade Nova de

Lisboa. Professora Associada, Universidade Nova de Lisboa,

Faculdade de Ciências e Tecnologia, Departamento

de Ciência e Engenharia do Ambiente, investigadora

no MARE NOVA - Centro de Ciência do Mar e do Ambiente, 2829-516 Caparica, Portugal.

Doctorada en Ingeniería Ambiental / Sistemas Sociales,

Facultad de Ciencias y Tecnología, Universidad Nueva

de Lisboa. Profesora Asociada, Universidad Nueva de

Lisboa, Facultad de Ciencias y Tecnología, Departamento

de Ciencias e Ingeniería Ambiental, investigadora en MARE

NOVA - Centro de Ciencias del Mar y del Medio Ambiente,

2829-516 Caparica, Portugal.

$\mathrm{PhD}$ in Environmental Engineering/Social Systems, Nova University of Lisbon, Faculty of Sciences and Technology.

Professor, Nova University of Lisbon, Faculty of Sciences and Technology, Department of Sciences and

Environmental Engineering, researcher at MARE - Marine and Environmental Sciences Centre, 2829-516 Caparica,

Portugal. 


\section{Diagnostic test of resilience potential

\author{
The radar of organizational resilience
}

ABSTRACT: This article drawn from a case study conducted between 2011 and 2015 explores the concept of potential resilience in the Portuguese Navy during the financial shock occurred in Portugal (2011). Theoretical and empirical research aimed at developing a potential diagnostic tool for capturing echoes resilience is designed to map resiliency potential echoes designated proto-resilience echoes. Results obtained indicate that the Radar-R has a set of non-intrusive benefits that enable actors in the system, an ambiguous environment, structuring thought clear and focused manner to identify enhancer levers for the organization respond strategically to change. This paper aims to stimulate the development of theoretical and empirical studies of resilience of organizations in scientific and organizational communities / business of Portuguese.

Keywords: Resilience; Assessment; Shock; Organization; Strategy

Benoît Robert

benoit.robert@polymtl.ca

Doutorado em Engenharia Civil, Polytechnique Montreal.

Professor, Polytechnique Montreal, Departamento de

Matemáticas e Engenharia Civil, Diretor do Centre Risque

\& Performance, Montreal, Quebeque, Canadá H3C 3A7.

Investiga e desenvolve uma metodologia de avaliação

de resiliência organizacional em organizações

governamentais e não-governamentais.

Doctorado en Ingeniería Civil, Polytechnique Montreal.

Profesor, Polytechnique Montreal, Departamento de

Matemáticas e Ingeniería Civil, Director del Centro Risque

\& Performance, Montreal, Quebec, Canadá H3C 3A7.

Investiga y desarrolla una metodología de evaluación

de la resiliencia organizacional en organizaciones

gubernamentales y no gubernamentales.

Phd in Civil engineering, Polytechnique Montreal.

Professor, Polytechnique Montréal, Mathematics and

Engineering Department, Director of the Centre Risque

\& Performance, Montréal, Quebec, Canada H3C 3A7.

Researches and develops in governmental

and non-governmental organization resilience

assessment methodology.

Recebido em abril de 2016 e aceite em outubro de 2016.

Recibido en abril de 2016 y aceptado en Octubre de 2016.

Received in April 2016 and accepted in October 2016. 
A incerteza e a imprevisibilidade de perturbações cada vez mais frequentes e atípicas estão a mobilizar a comunidade científica, política e empresarial para explorarem novos paradigmas e instrumentos organizacionais centrados no conceito de resiliência.

Este trabalho de investigação exploratório, desenvolvido na Marinha portuguesa, baseia-se nos trabalhos de investigação do Centre Risque \& Performance, de Montreal, no Canadá, e procura colmatar uma lacuna identificada na literatura em 2011, designadamente, a ausência de uma abordagem orientada para o mapeamento da resiliência potencial de uma organização num período de choque.

A pertinência desta problemática emergiu na sequência de um choque orçamental, ocorrido em Portugal (2011). Tendo em conta a natureza deste fenómeno contemporâneo, analisado em contexto real, e a questão de investigação (como?), optou-se por um modelo de investigação do tipo estudo de caso (Yin, 1994), orientado para a investigação-ação. O objetivo desta investigação é desenvolver um instrumento de diagnóstico que permita mapear as coordenadas da resiliência potencial por captação de ecos, durante um período de choque.

$\mathrm{Na}$ primeira parte deste artigo, procede-se à revisão bibliográfica realizada fundamentalmente até 2012. De seguida, faz-se uma breve análise do estudo de caso e, nas secções seguintes, apresentam-se os elementos teóricos e empíricos que serviram de base ao desenvolvimento do instrumento. Segue-se uma breve descrição da sua aplicação e a metodologia de avaliação. Finalmente, nas duas últimas secções, mostram-se os resultados e tece-se a conclusão.

À semelhança de outros países, as organizações dos países de língua portuguesa enfrentam um conjunto de eventos adversos que põem em causa o equilíbrio estrutural das suas respetivas organizações.

Tendo em conta a relevância da temática e a escassez de publicações sobre resiliência em língua portuguesa, com este artigo pretende-se contribuir para estimular o debate e a apropriação do conceito nas comunidades científicas e organizacionais/empresariais.

\section{Referencial teórico}

\section{Breve histórico}

No âmbito da ciência moderna, até aos nossos dias, o conceito de resiliência evoluiu devido a sucessivos alargamentos científicos, dando origem a um conceito transversal e polissémico.

Seja na área dos materiais (Charmet, 1980; Timoshenko, 1983; Carneiro, 1999; Peneireiro, 2010), ou no âmbito da psicologia (Werner, 1993; Rutter, 1993; Luthar, 2000), da ecologia (Holling, 1973), da socioecologia (Holling, 2001; Gunderson, Holling, 2001; Carpenter, 2001; Folke et al., 2002), da Estratégia Internacional de Redução de catástrofes (UN/ISDR, 2005-2015), dos sistemas industriais (Hollnagel, 2006, 2012), das infraestruturas críticas

\section{O objetivo desta investigação é desenvolver um instrumento de diagnóstico que permita mapear as coordenadas da resiliência potencial por captação de ecos, durante um período de choque.}


(Robert et al., 2012; Petit et al., 20012) e das políticas públicas de segurança e de competitividade económica (The Reform Institute, 2008; Ministére de la Sécurité Publique, Canadá, 2008; Australia Strategic Policy Institute, 2008; White House, 2011), o conceito de resiliência tem granjeado um enorme sucesso enquanto fator de segurança e de sustentabilidade das organizações.

\section{Conceitos atuais de resiliência das organizações}

Neste artigo consideramos que a emergência da resiliência das organizações resultou da evolução do pensamento internacional, em matéria de redução de riscos de catástrofes. Com efeito, a Década Internacional para a Redução de Catástrofes Naturais (1990-1999) mobilizou de tal forma a comunidade internacional que foi criada a Estratégia Internacional de Redução de Catástrofe (2000) e, em 2005, foi aprovado o Quadro de Ação de Hyogo (2005-2015), com o objetivo de construir a resiliência das nações e das organizações face aos riscos de catástrofes.

No âmbito do UN/International Strategy for Disaster Reduction (ISDR), a resiliência é definida como "a capacidade de um sistema organizacional, uma comunidade ou uma sociedade exposta a riscos, suportar, absorver, acolher e corrigir os efeitos de um perigo, em tempo oportuno e de maneira eficaz, nomeadamente pela preservação e pelo restabelecimento das suas estruturas essenciais e das suas funções básicas» (Terminology on Disaster Risk Reduction, 2009, p. 24).

Embora o movimento iniciado em Hyogo estivesse orientado para responder às catástrofes, alguns países assumiram uma visão mais audaz e abrangente ao integrarem, nas suas respetivas políticas públicas, a construção da resiliência enquanto desígnio nacional, alargado à esfera da segurança e da economia (EUA, 2008; Austrália, 2008; Canadá, 2008). Neste contexto, a resiliência emerge como uma resposta adaptativa a um conjunto de eventos adversos. Alguns autores e organizações já vinham a alertar para o facto de os modelos existentes estarem a tornar-se ineficazes para assegurar a segurança e a competitividade económica da sociedade (Richmond, 2003; Dauphiné, Provitolo, 2007; Hollnagel, 2012; Robert et al., 2012).

\section{Principais pesquisas sobre o tema}

Embora atualmente seja difícil avaliar quais são as principais pesquisas sobre resiliência, alguns trabalhos de investigação teóricos e empíricos evoluíram para a conceção de instrumentos de avaliação desenvolvidos em três conceituados centros de investigação: ANL norte-americano, CRC francês e CRP canadiano.

O Argonne National Laboratory (ANL) é um centro de pesquisa do Departamento de Energia dos Estados Unidos, no qual se desenvolveu, em parceria com o Department of Homeland Security, um conjunto de índices de maneira a identificar a resiliência nas infraestruturas críticas da economia, da sociedade civil, da governança (serviços de urgência), das
Alguns autores e organizações já vinham a alertar para o facto de os modelos existentes estarem a tornar-se ineficazes para assegurar a segurança e a competitividade económica da sociedade. 
cadeias de aprovisionamento, etc. Definindo resiliência como «a capacidade de uma entidade, uma organização, uma comunidade ou uma região em antecipar, resistir, absorver, responder, adaptar-se e recuperar depois de um distúrbio» (Carlson, L., et al., 2012; Petit, Eaton, Fisher, 2012), os trabalhos desenvolvidos visam reforçar a preparação (antecipação), as medidas de redução (resistência e absorção), a capacidade de resposta (resposta e adaptação) e mecanismos de restabelecimento.

O Centre de Recherche sur les Risques et les Crises (CRC) é um laboratório interdisciplinar da Escola Nacional Superior de Minas de Paris (MINES ParisTech), que está associado a uma cadeira designada por «Engenharia da Resiliência» desenvolvida por Eric Hollnagel. Este autor define Engenharia da Resiliência como "a aptidão intrínseca de um sistema ajustar o seu funcionamento antes, durante e após a ocorrência de uma perturbação ou de uma mudança significativa, de modo a que possa continuar a exercer a sua atividade em condições esperadas ou inesperadas» (Hollnagel, 2012, prologue, xxxvi).

De acordo com Nemeth et al. (2009), os trabalhos desenvolvidos no âmbito da Engenharia da Resiliência assentam nas seguintes hipóteses teóricas:

- Os indivíduos de uma organização devem sistematicamente ajustar os seus comportamentos à situação e aos recursos disponíveis;

- Algumas situações podem ser atribuídas a falhas ou a disfuncionalidades. As situações anormais podem ser estudadas como a consequência de combinações inesperadas da variabilidade dos comportamentos dos sistemas;

- A gestão da segurança não pode ser apenas baseada em eventos do passado e na avaliação da probabilidade da ocorrência de falhas. Deve ser também baseada em comportamentos proativos;

- A gestão da segurança não pode ser abordada isoladamente das outras funções da gestão das organizações.

Para Rigaud (2011), a Engenharia da Resiliência representa uma nova geração de segurança funcional para as organizações.

O Centre Risque \& Performance (CRP) é o centro de investigação integrado no Departamento de Matemática e Engenharia da École Polytechnique de Montréal (Quebeque - Canadá). Orientado para uma abordagem por consequências, os trabalhos desenvolvidos pelo CRP estão direcionados para o estudo das interdependências das infraestruturas críticas. O CRP desenvolveu junto dos sistemas essenciais do Quebeque, uma forma de avaliar a resiliência, baseada na conceção de um guia metodológico (Pinel, 2009; Pairet, 2009) designado por Résilience Organisationnelle - Concept et Méthodologie d'Évaluation. Nesta metodologia, a resiliência é definida como «a capacidade de um sistema manter ou restabelecer níveis de funcionamento aceitáveis apesar das perturbações e das falhas» (Pinel, 2009, p. 71). 
Na sua versão inicial (2011), data em que a metodologia foi parametrizada à Marinha portuguesa, o modelo era constituído por quatro etapas: (1) Perfil do sistema; (2) Estudo dos recursos utilizados e fornecidos; (3) Gestão das falhas; e (4) Avaliação da resiliência. Entretanto, o modelo tem evoluído para conceções relacionadas com o potencial de resiliência Hémond (2013) e com as Análises Diagnósticas de Resiliência (Robert et al., 2014).

\section{Síntese conceitual do trabalho}

Este trabalho de investigação, orientado para a criação de um instrumento diagnóstico, permite mapear as coordenadas de resiliência potencial por captação de ecos e nele se apresenta uma fundamentação teórica e empírica diversificada, que passamos a descrever.

Relativamente à resiliência potencial, consideramos que o conceito encontra a sua sustentação teórica no ciclo de adaptação dos sistemas socioecológicos desenvolvidos por Holling (2001). Embora o autor não utilize especificamente o termo «resiliência potencial», ele considera que, quando um sistema sofre um choque e ultrapassa a sua resiliência, liberta recursos que foram acumulados anteriormente. Desta forma, dão origem à mobilização do potencial interno, de modo a gerar a inovação de um novo ciclo, caso os recursos sejam suficientes, ou colapsar, caso os recursos não sejam bastantes para responder à disrupção.

Neste trabalho, apresenta-se paralelamente um cruzamento dos trabalhos teóricos e empíricos, desenvolvidos pelo CRP, nomeadamente os baseados nos conceitos provenientes do modelo de Avaliação de Resiliência Organizacional (Pinel, 2009; Pairet, 2009) e o trabalho desenvolvido por Ribeiro (2008). Estes trabalhos são desenvolvidos no âmbito do modelo de gestão estratégica, relativamente aos paradigmas de transformação, que permitem assegurar a coerência e a adaptação de uma organização, e são aplicados à Marinha.

\section{Estrutura de um instrumento de diagnóstico de resiliência potencial por captação de ecos \\ Estrutura do Radar da Resiliência (Radar-R)}

Decorrente das influências teóricas e empíricas supracitadas, foi concebido um instrumento de avaliação diagnóstica de resiliência potencial por captação de ecos, designado por Radar da Resiliência (Radar-R), que permite mapear as coordenadas da estrutura de uma organização.

Tendo em conta que os choques representam eventos disruptivos de alta intensidade, considera-se que a avaliação diagnóstica de resiliência potencial deve ser objeto de uma representação e aplicação simplificada e pouco intrusiva, de modo a facilitar a aplicação na organização (ver Figura 1, p. 51).

Do ponto de vista estrutural, passamos a descrever os conceitos que estiveram na base da formação gráfica do modelo. O Radar-R é composto 


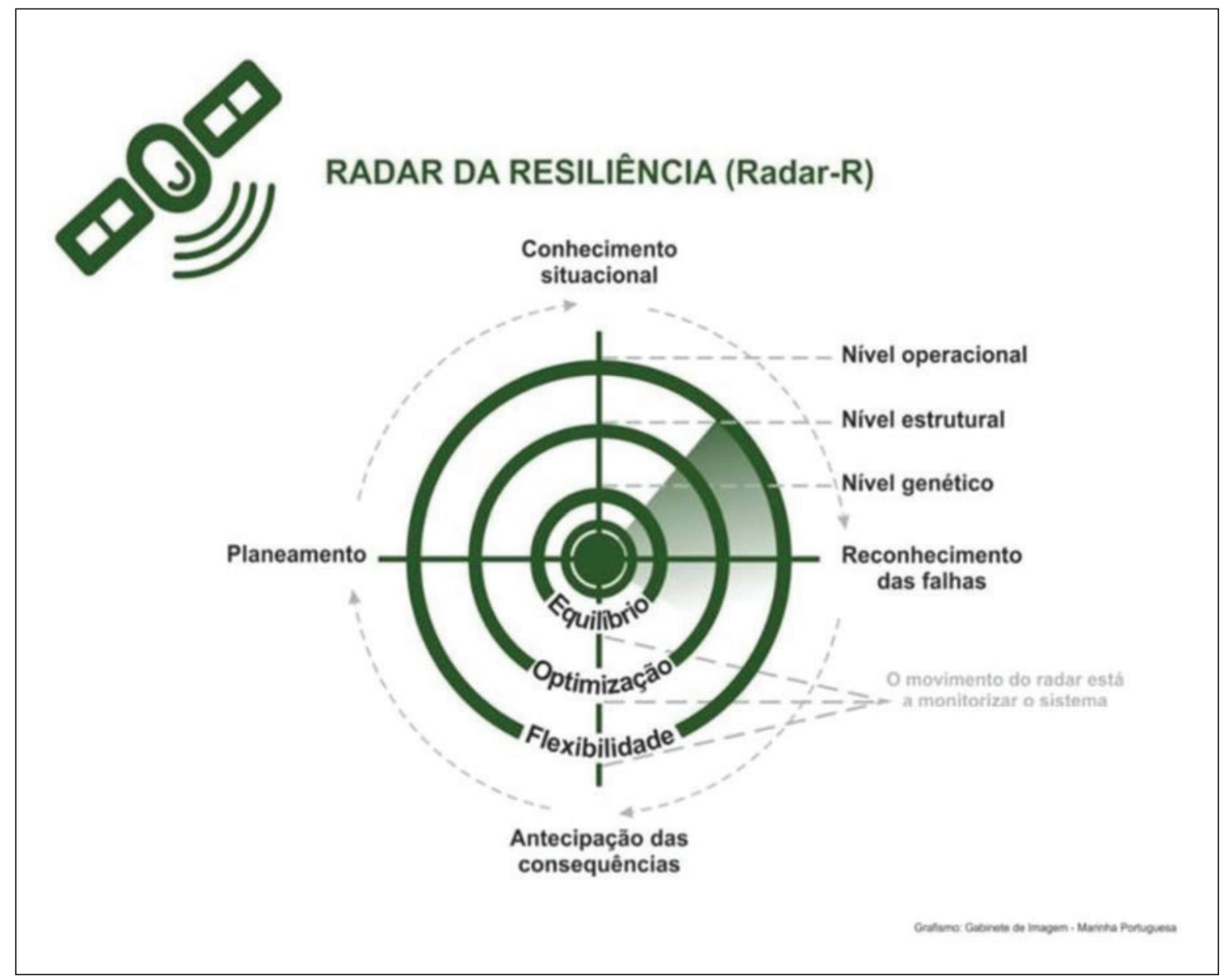

por quatro eixos que representam as quatro dimensões da Metodologia de Avaliação de Resiliência Organizacional desenvolvida pelo CRP. Para além disso, integra três círculos que mostram os três níveis sistémicos de uma organização e, simultaneamente, três paradigmas de transformação desenvolvidos por Ribeiro (2007).

De acordo com a Figura 1, os quatro eixos do Radar-R representam as seguintes dimensões que permitem garantir a resiliência de uma organização: (1) o conhecimento; (2) o reconhecimento; (3) a antecipação; e (4) a planificação.

As características destas quatro dimensões são as seguintes:

1. O conhecimento (awareness, no original em inglês): o conhecimento da situação é a primeira dimensão. Trata-se de sondar a organização para apurar a qualidade da informação que ela possui sobre os seus ambientes interno e externo. Esta dimensão, para além de inúmeras vantagens, permite identificar a coerência dos setores no interior da organização;

2. O reconhecimento: o reconhecimento das falhas, erros e disrupções é a 
segunda dimensão. Partindo do princípio que uma situação apenas existe quando é reconhecida, esta dimensão permite à organização definir estados de funcionamento aceitáveis, a partir de diferentes estados de referência: estado normal, estado degradado, ou estado de falha. É a partir desta dimensão que uma organização pode lançar aos setores estados de alerta, de modo a criar medidas de flexibilidade, mediante uma vasta gama de alternativas;

3. A antecipação: a antecipação das consequências sobre os territórios e as suas populações constitui a terceira dimensão. Está relacionada com um conjunto de medidas preventivas, corretivas ou de proteção que permitem antecipar possíveis perturbações a nível interno e do ambiente externo. A antecipação possibilita definir margens de manobra necessárias à manutenção do funcionamento aceitável e a um retorno à normalidade, ou ainda, quando o choque tem uma intensidade superior aos recursos disponíveis, viabiliza a restruturação do sistema organizacional;

4. A planificação: a planificação é a quarta dimensão. Permite integrar um conjunto de orientações estratégicas, de objetivos e de programas de ação que vão dar origem a um novo conhecimento de lições aprendidas.

Para completar a representação, o Radar-R é constituído por três círculos que representam o corte simples de uma organização, concetualizada em três níveis sistémicos: (1) input; (2) sistema; e (3) output.

As características dos três níveis são as seguintes:

1. O input: $\mathrm{O}$ input representa todos os recursos humanos, financeiros, matérias-primas, tecnologia, informação, etc., que entram no sistema organizacional e que são necessários ao fornecimento de um determinado produto ou serviço à população, ou a outros sistemas organizacionais;

2. O sistema: O sistema é composto de subsistemas e de um processo, correspondentes ao conjunto das atividades da organização que labora a produzir produtos/serviços;

3. O output: O output corresponde aos produtos ou serviços fornecidos pela organização ao ambiente, sejam eles clientes ou população, no caso de serviços públicos.

Enquanto qualidade intrínseca do sistema, consideramos que a resiliência é composta por três propriedades, identificadas nos paradigmas de transformação e aplicados à Marinha (Ribeiro, 2008; Documentação estruturante da Marinha, 2011). Como podemos observar na Figura 1, cada círculo corresponde às três propriedades que permitem caracterizar a resiliência: (1) equilíbrio; (2) otimização; e (3) flexibilidade.

As características das três propriedades são as seguintes:

1. O equilíbrio: ao nível do input, o equilíbrio representa a distribuição proporcional de todos os recursos disponíveis, através de um fluxo de entrada orientado para a diversidade de respostas. O equilíbrio é um estado que garante a eficiência do sistema e é através dele que se estabelecem as diretrizes para criar as condições mais favoráveis à otimização;
A protorresiliência é um neologismo, que criámos no âmbito do nosso trabalho, para especificar o conceito de resiliência potencial por captação de ecos e que permite uma maior maleabilidade para trabalhar os dados. 
Tabela 1 Estrutura esquemática do questionário

\begin{tabular}{|c|c|c|c|}
\hline \multirow{3}{*}{ Categorias do Questionário } & \multicolumn{3}{|c|}{ Nível sistémico da organização } \\
\hline & Input & Sistema & Output \\
\hline & $\begin{array}{l}\text { Nível recursos } \\
\text { (genético) }\end{array}$ & $\begin{array}{l}\text { Nível estrutural } \\
\text { (estrutura) }\end{array}$ & $\begin{array}{l}\text { Nível operacional } \\
\text { (produto) }\end{array}$ \\
\hline \multicolumn{4}{|l|}{ Conhecimento situacional } \\
\hline \multicolumn{4}{|l|}{ Reconhecimento } \\
\hline \multicolumn{4}{|l|}{ Antecipação das consequências } \\
\hline Planeamento & & & \\
\hline
\end{tabular}

2. A otimização: ao nível do sistema, a otimização consiste na realização de processos e ações que promovam uma eficiência dos recursos, por forma a produzir o melhor rendimento possível no sistema;

3. A flexibilidade: ao nível do output, a flexibilidade representa a diversidade de respostas adaptativas produzidas pelo sistema.

\section{Inquérito por questionário grelha}

O Radar-R é operacionalizado através de um inquérito por questionário que integra a sua configuração. $\mathrm{O}$ inquérito é composto por quatro perguntas semiabertas, relacionadas com as dimensões da resiliência: (1) conhecimento; (2) reconhecimento; (3) antecipação; e (4) planificação, nos três níveis sistémicos: (1) input; (2) sistema; e (3) output, onde se procura identificar ecos de resiliência potencial: (1) o equilíbrio; (2) a otimização; e a (3) a flexibilidade (ver Tabela 1), perfazendo um total de 12 perguntas por setor.

O objetivo do questionário é localizar ecos informativos de resiliência potencial na estrutura da organização, os quais designamos por ecos de protorresiliência. A protorresiliência é um neologismo, que criámos no âmbito do nosso trabalho, para especificar o conceito de resiliência potencial por captação de ecos e que permite uma maior maleabilidade para trabalhar os dados. Este novo conceito é composto pelo prefixo de origem grega "proto", que significa início, começo, anterioridade, e pelo substantivo "resiliência», que significa a capacidade de o sistema acolher o impacto de um choque. 


\section{Estudo de caso: a Marinha portuguesa}

\section{Enquadramento do estudo de caso}

A primeira fase da investigação correspondeu à parametrização e aplicação à Marinha portuguesa do modelo desenvolvido pelo CRP, com o objetivo de avaliar a resiliência da Marinha, orientada para o apoio às populações ribeirinhas, em caso de catástrofe.

Todavia, durante a aplicação do modelo, ocorreu em Portugal um choque orçamental que obrigou o país a recorrer a uma ajuda externa do FMI, provocando uma forte turbulência em inúmeros setores da vida social portuguesa. Nestas circunstâncias, o nosso projeto de investigação foi reformulado, por forma a explorar o conceito de resiliência potencial durante um período de choque.

\section{Dimensão do território português}

Portugal é constituído por um território terrestre de cerca de $91000 \mathrm{~km}^{2}$, com $848 \mathrm{~km}$ de comprimento e uma largura de $250 \mathrm{~km}$. A identidade de Portugal, enquanto nação marítima, encontra a sua justificação na dimensão da sua orla costeira. O território marítimo português representa uma área da responsabilidade da Marinha portuguesa, tendo uma área territorial de cerca de $3858431 \mathrm{~km}^{2}$, ou seja, cerca de 42 vezes o território nacional terrestre (ver Figura 2).

\section{O modelo de Marinha de duplo uso}

$\mathrm{Na}$ Marinha portuguesa foi desenvolvido um conceito de «Marinha de duplo uso» (Monteiro, 2010; Documentação oficial da Marinha, 2011; Canadian Coast Guard, 2012). Este conceito foi baseado num modelo histó-

Figura 2 Espaço marítimo de Portugal

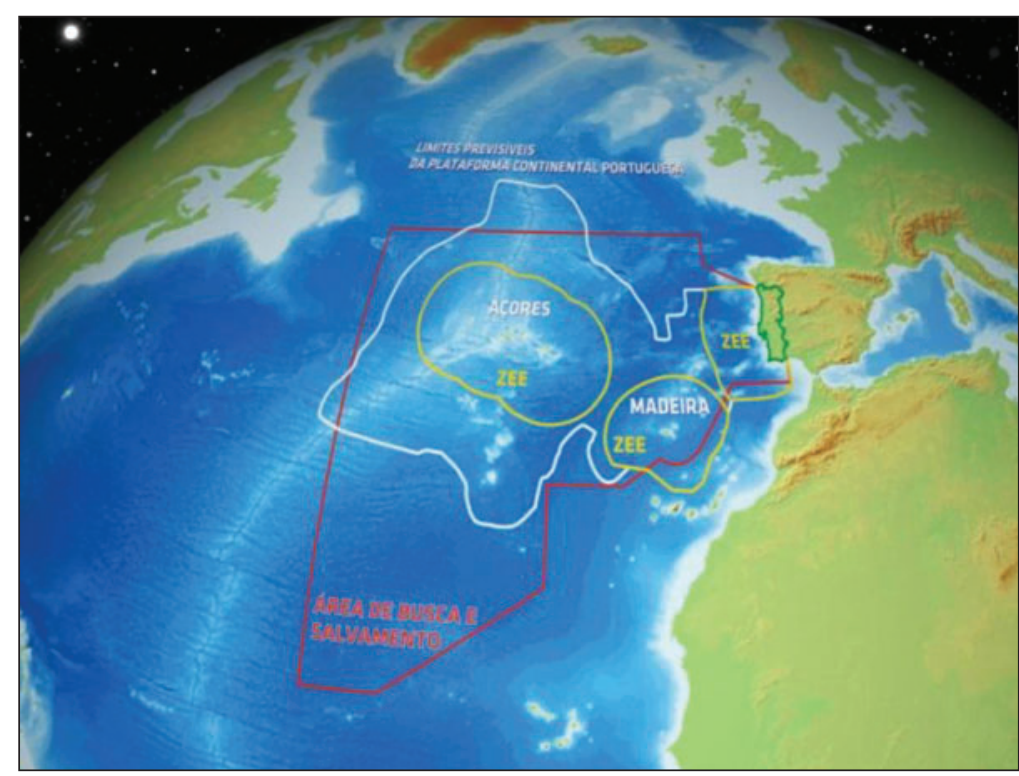


rico-cultural português herdado dos monarcas portugueses, que acreditavam que, sem duplicar os dispendiosos recursos de uma armada e de uma guarda costeira, seria possível garantir a proteção, a segurança e o desenvolvimento dos espaços marítimos de Portugal.

No período do choque orçamental, em que um dos seus maiores impactos na Marinha foi a cisão da Autoridade Marítima Nacional (2014), na primeira etapa do modelo de avaliação de resiliência organizacional, foi traçado o perfil da «Marinha de duplo uso» (ver Figura 3).

O sistema «Marinha de duplo uso» é um sistema organizacional orientado para a racionalização dos recursos, a interdependência alinhada e a coerência, e é composto por três níveis sistémicos: nível do fluxo de entrada, sistema, e fluxo de saída. Ao nível do fluxo de entrada, identificamos um conjunto de condicionalismos externos (a conjuntura nacional e internacional, a vontade política e a legislação) e internos (orgânica, estratégia naval, liderança, vontade e crenças dos dirigentes) que influenciam o sistema. Ao nível do sistema, esta organização é constituída por três pilares estratégicos de duplo uso que sustentam três funções estratégicas, constituídas por três tarefas essenciais.

Trata-se de uma estrutura piramidal, cuja visão foi herdada do passado e

Figura 3 Quadro interpretativo da Marinha de duplo uso

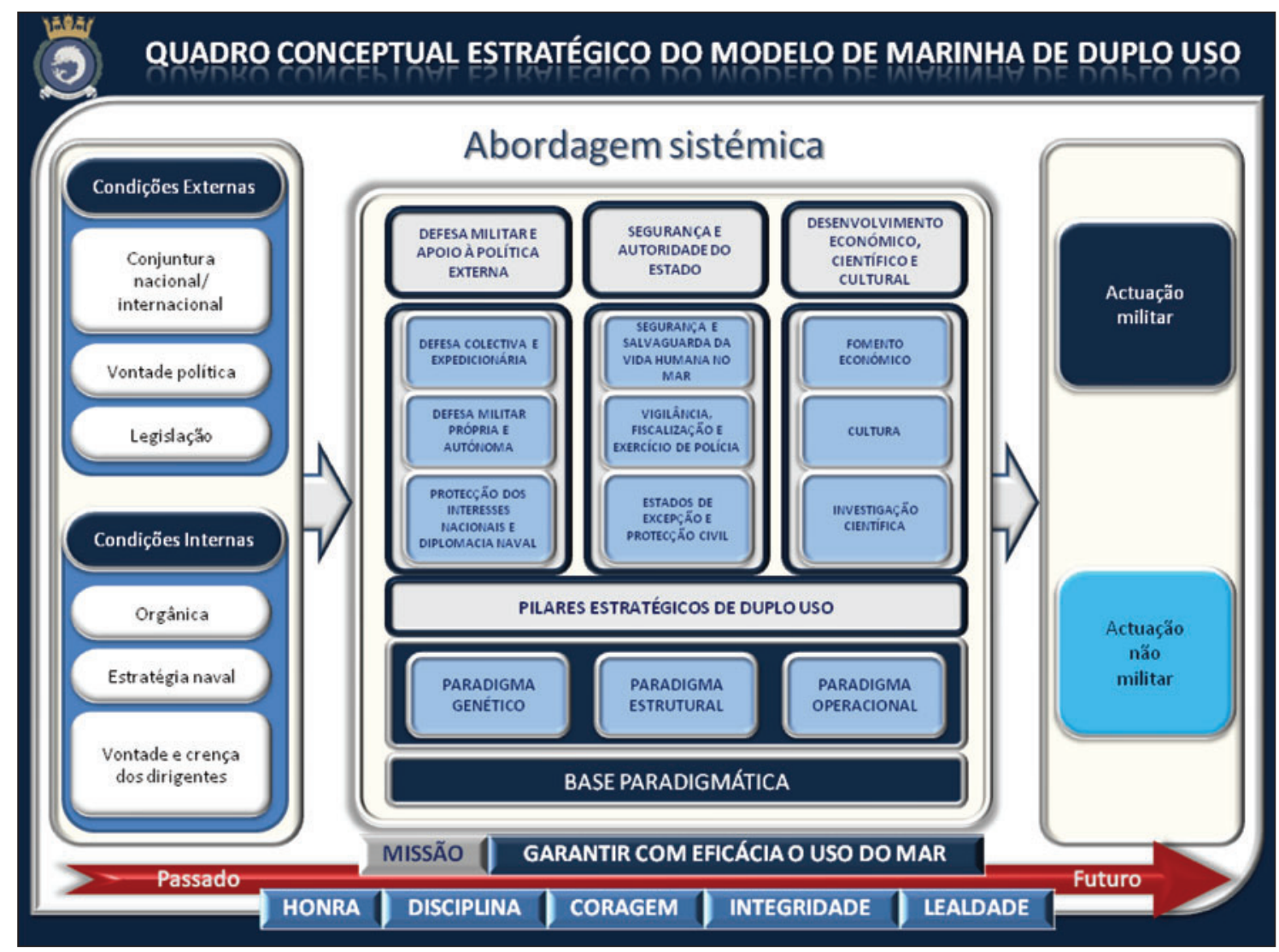


está orientada para o futuro através dos paradigmas de transformação que visam a adaptação da Marinha aos desafios do futuro.

Ao nível do fluxo de saída, a Marinha portuguesa oferece ao Estado e às populações um conjunto de produtos/serviços de natureza militar e não militar que permitem garantir o uso do mar. Os valores traduzidos nos símbolos heráldicos garantem a verticalidade e a coesão de todos os elementos do sistema para cumprirem e garantirem o cumprimento da missão, de modo a estabelecer com eficácia o uso do mar.

\section{Aplicação do instrumento}

O processo de aplicação do instrumento foi acompanhado pela supervisão do chefe da Divisão de Planeamento (DIVPLAN). As entrevistas semidiretivas foram realizadas com os responsáveis de cada setor, de acordo com o guião que incluía duas partes essenciais: (1) enquadramento do trabalho de investigação focado no conceito de resiliência e (2) apresentação do Radar-R.

Ficou ao critério do responsável de cada setor a recolha de dados, sendo acordado que as respostas aos questionários seriam enviadas para o endereço eletrónico do Oficial de Ligação.

Procedeu-se a três fases de aplicação do Radar-R. Na 1. a fase de aplicação, foram inquiridos os setores da Marinha, conforme a informação descritiva no Anexo à Diretiva de Política Naval, n. ${ }^{\circ}$ 025/DIVPLAN, 03OUT11: a Superintendência dos Serviços do Pessoal (SSP); a Superintendência dos Serviços Financeiros (SSF); a Superintendência dos Serviços de Tecnologia da Informação (SSTI); a Superintendência dos Serviços do Material; Escola Naval (EN); Comissão Cultural da Marinha (CCM); Direção-Geral da Autoridade Marítima (DGAM); Comando Naval (CN); e Instituto Hidrográfico (IH).

Após a recolha dos primeiros dados, foi decidido ampliar o Radar-R aos setores operacionais, isto é, aos meios navais constituídos pela Flotilha, esquadra de navios oceânicos, esquadra de navios de patrulha e unidade de mergulhadores. Apesar do Estado-Maior da Armada (EMA) não ser considerado um setor, foi igualmente inquirido pela sua relevância, enquanto gabinete de aconselhamento do Chefe do Estado-Maior da Armada (CEMA).

$\mathrm{Na} 2 .^{\mathrm{a}}$ fase, os resultados foram apresentados à equipa de investigação do CRP em Montreal. Apesar da componente concetual e estrutural ter sido reconhecida como coerente, a metodologia de avaliação do Radar-R carecia de maior desenvolvimento. $\mathrm{Na} 3 .^{\mathrm{a}}$ fase, foi aplicado o Radar-R à unidade de Fuzileiros.

\section{Metodologia de avaliação diagnóstica do Radar-R \\ Instrumentos de recolha de dados e análise por captação de ecos}

Para proceder à avaliação diagnóstica, foi criado um conjunto de 7 instrumentos de análise que permitem analisar os dados de uma forma organizada: 
1. Grelha matriz: é uma tabela base, constituída pelos oito setores da Marinha identificados por letras (A, B, C, D, E, F, G, H) e composta pelas quatro dimensões da resiliência e os três níveis sistémicos. Consideramos a grelha matriz como a representação estilizada do território concetual, constituído por 96 espaços, segmentados em 12 respostas por setor;

2. Grelha de coordenadas (ver Tabela 2): é uma grelha de referência, mapeada com as coordenadas constituídas pelos setores em análise. Esta grelha representa o mapa do território concetual da organização, constituída por oito setores, perfazendo um total de 96 coordenadas (32 coordenadas por propriedades, uma vez que os indicadores não possuem coordenadas). A leitura da coordenada é feita da seguinte forma: Letra:

Tabela 2 Grelha de coordenadas

\begin{tabular}{|c|c|c|c|c|c|c|c|c|c|c|}
\hline \multirow{2}{*}{\multicolumn{2}{|c|}{$\begin{array}{l}\text { Dimensões } \\
\text { da } \\
\text { Resiliência }\end{array}$}} & \multirow{2}{*}{$\begin{array}{l}\text { Níveis } \\
\text { Sistémicos }\end{array}$} & \multicolumn{8}{|c|}{ Setores da Marinha de duplo uso } \\
\hline & & & A & B & C & $\mathrm{D}$ & $\mathrm{E}$ & $\mathrm{F}$ & G & $\mathrm{H}$ \\
\hline \multirow{4}{*}{ 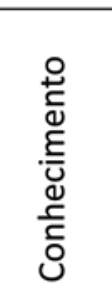 } & \multirow{4}{*}{$\Xi$} & Processos & & & & & & & & \\
\hline & & Genético (1) & A.1.1 & B.1.1 & C.1.1 & D.1.1 & E.1.1 & F.1.1 & G.1.1 & H.1.1 \\
\hline & & Estrutural (2) & A.1.2 & B.1.2 & C.1.2 & D.1.2 & E.1.2 & F.1.2 & G.1.2 & H.1.2 \\
\hline & & Operacional (3) & A.1.3 & B.1.3 & C.1.3 & D.1.3 & E.1.3 & F.1.3 & G.1.3 & H.1.3 \\
\hline \multirow{4}{*}{ 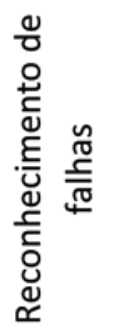 } & \multirow{4}{*}{$\bar{d}$} & Mecanismos & & & & & & & & \\
\hline & & Genético (1) & A.2.1 & B.2.1 & C.2.1 & D.2.1 & E.2.1 & F.2.1 & G.2.1 & H.2.1 \\
\hline & & Estrutural (2) & A.2.2 & B.2.2 & C.2.2 & D.2.2 & E.2.2 & F.2.2 & G.2.2 & H.2.2 \\
\hline & & Operacional (3) & A.2.3 & B.2.3 & C.2.3 & D.2.3 & E.2.3 & F.2.3 & G.2.3 & H.2.3 \\
\hline \multirow{4}{*}{ 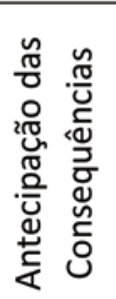 } & \multirow{4}{*}{$\bar{m}$} & Instrumentos & & & & & & & & \\
\hline & & Genético (1) & A.3.1 & B.3.1 & C.3.1 & D.3.1 & E.3.1 & F.3.1 & G.3.1 & H.3.1 \\
\hline & & Estrutural (2) & A.3.2 & B.3.2 & C.3.2 & D.3.2 & E.3.2 & F.3.2 & G.3.2 & H.3.2 \\
\hline & & Operacional (3) & A.3.3 & B.3.3 & C.3.3 & D.3.3 & E.3.3 & F.3.3 & G.3.3 & H.3.3 \\
\hline \multirow{4}{*}{ 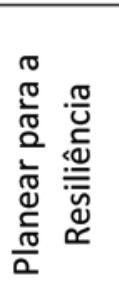 } & & Contributos & & & & & & & & \\
\hline & & Genético (1) & A.4.1 & B.4.1 & C.4.1 & D.4.1 & E.4.1 & F.4.1 & G.4.1 & H.4.1 \\
\hline & & Estrutural (2) & A.4.2 & B.4.2 & C.4.2 & D.4.2 & E.4.2 & F.4.2 & G.4.2 & H.4.2 \\
\hline & & Operacional (3) & A.4.3 & B.4.3 & C.4.3 & D.4.3 & E.4.3 & F.4.3 & G.4.3 & H.4.3 \\
\hline
\end{tabular}


representa o setor $(\mathrm{A}, \mathrm{B}, \mathrm{C}, \mathrm{D}, \mathrm{E}, \mathrm{F}, \mathrm{G}, \mathrm{H})$; o $1 .^{\circ}$ número representa a dimensão $(1,2,3,4)$; e o $2 .^{\circ}$ número representa o nível sistémico $(1,2,3)$;

3. Tabela de critérios: é constituída por um conjunto de critérios associados a uma notação simbólica para identificar os ecos;

4. Grelha de triagem: permite compilar os dados recolhidos a partir dos inquéritos;

5. Grelha de mapeamento: contém a informação das dimensões e dos ecos das propriedades de protorresiliência, devidamente organizados nos níveis e nos espaços apropriados;

6. Gráfico circular de coordenadas (ver Gráfico 1): corresponde à grelha das coordenadas com uma configuração circular;

7. Gráfico circular de mapeamento de ecos: corresponde à representação final, onde podemos identificar, através de símbolos, os ecos de protorresiliência dos setores mapeados nos respetivos níveis. Neste gráfico não são identificadas explicitamente as dimensões, uma vez que elas representam o espaço onde se manifestam os ecos.

\section{Breve descrição do procedimento}

Depois de identificar os instrumentos, procedemos à descrição das várias fases de análise de dados, tendo em conta cinco fases que passamos a descrever:

Gráfico 1 Coordenadas

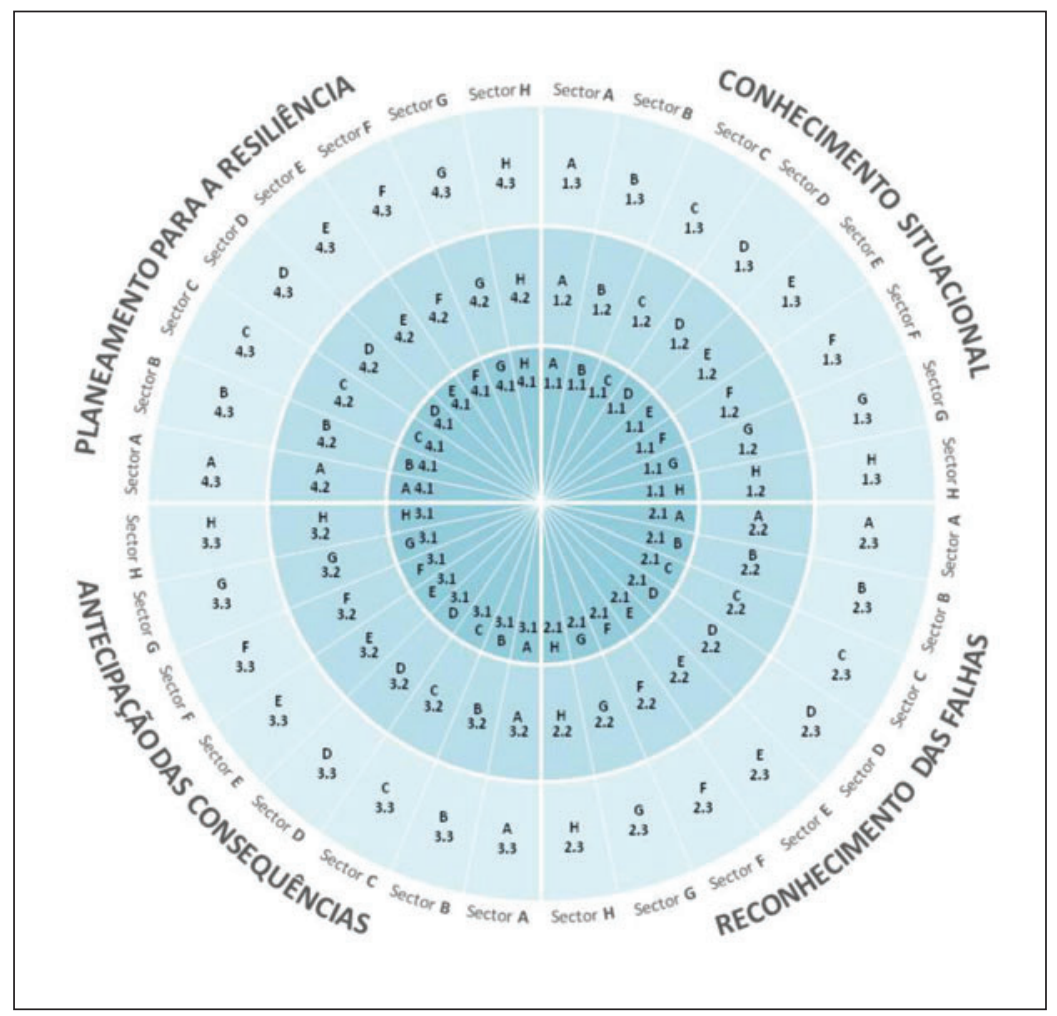


- Fase 1 - Aplicação do inquérito: esta fase consiste em aplicar o inquérito por questionário aos setores selecionados da organização;

- Fase 2 - Triagem: após inserir as respostas dos setores na grelha de triagem, procede-se à triagem dos ecos, através de uma análise de conteúdo. A triagem consiste em identificar nas respostas recolhidas dos setores a presença das quatro dimensões da resiliência e ecos das três propriedades, de acordo com a tabela de critérios;

- Fase 3 - Reagrupamento: esta fase consiste em reagrupar os indicadores e os ecos nas respetivas coordenadas da grelha de mapeamento;

- Fase 4-Transferência dos dados para o gráfico: os dados quantitativos da grelha de mapeamento relativos aos ecos de propriedades são transferidos para um gráfico circular;

- Fase 5 - Análise quantitativa dos indicadores e ecos: em termos gerais, a análise pode ser realizada através de percentagens, gráficos de barras, etc. No âmbito deste trabalho, optou-se por analisar os dados representados em gráficos circulares, gráficos de barras verticais e respetivas médias.

\section{Análise de dados}

Com base na pergunta de partida, orientada para a possibilidade de ser mapeada a resiliência potencial dos setores da Marinha num período de choque, os resultados alcançados, através do diagnóstico efetuado aos setores, confirmam que as propriedades de protorresiliência (o equilíbrio, a otimização e a flexibilidade) e as quatro dimensões da resiliência (conhecimento, reconhecimento, antecipação e planeamento) podem ser mapeadas, nos três níveis sistémicos (input, sistema, output) de uma organização, tal como apresentamos no Gráfico 2 (p. 60).

Numa primeira análise, o Gráfico 2 permite identificar os pontos fortes e as lacunas dos setores, através da identificação das coordenadas «cheias»e «vazias». Refere-se que, nesta fase da investigação, os símbolos estão colocados no espaço relativo à dimensão e ao setor.

Podemos observar que, durante o choque, o equilíbrio e a otimização foram as propriedades dominantes nas dimensões dos setores, devido à grande atividade da Marinha para responder ao choque. Em relação às lacunas, as 14 coordenadas sem ecos, ao nível do output, mostram como o estrangulamento orçamental reduziu a flexibilidade do produto da Marinha.

Os resultados permitiram efetuar várias análises diagnósticas. Para uma análise comparativa complementar, os dados recolhidos foram organizados em quatro gráficos de barras para mais facilmente se poder identificar, nos setores, as três propriedades presentes em cada dimensão.

\section{Dimensão do conhecimento}

O Gráfico 3 (p. 60) permite identificar que, na dimensão do conhecimento, o equilíbrio é a propriedade que apresenta valores com maior discrepância, sendo os valores mais elevados os do setor H (10 ecos), do setor D ( 9 ecos) e do setor

\author{
Durante o choque, \\ o equilíbrio \\ e a otimização foram \\ as propriedades \\ dominantes \\ nas dimensões \\ dos setores, devido \\ à grande atividade \\ da Marinha para \\ responder ao choque. \\ Em relação às lacunas, \\ as 14 coordenadas sem \\ ecos, ao nível do output, \\ mostram como \\ o estrangulamento \\ orçamental reduziu \\ a flexibilidade \\ do produto da Marinha.
}


$60 \mid$ Revista de GESTÃO dos Países de Língua Portuguesa

Gráfico 2 Ecos mapeados

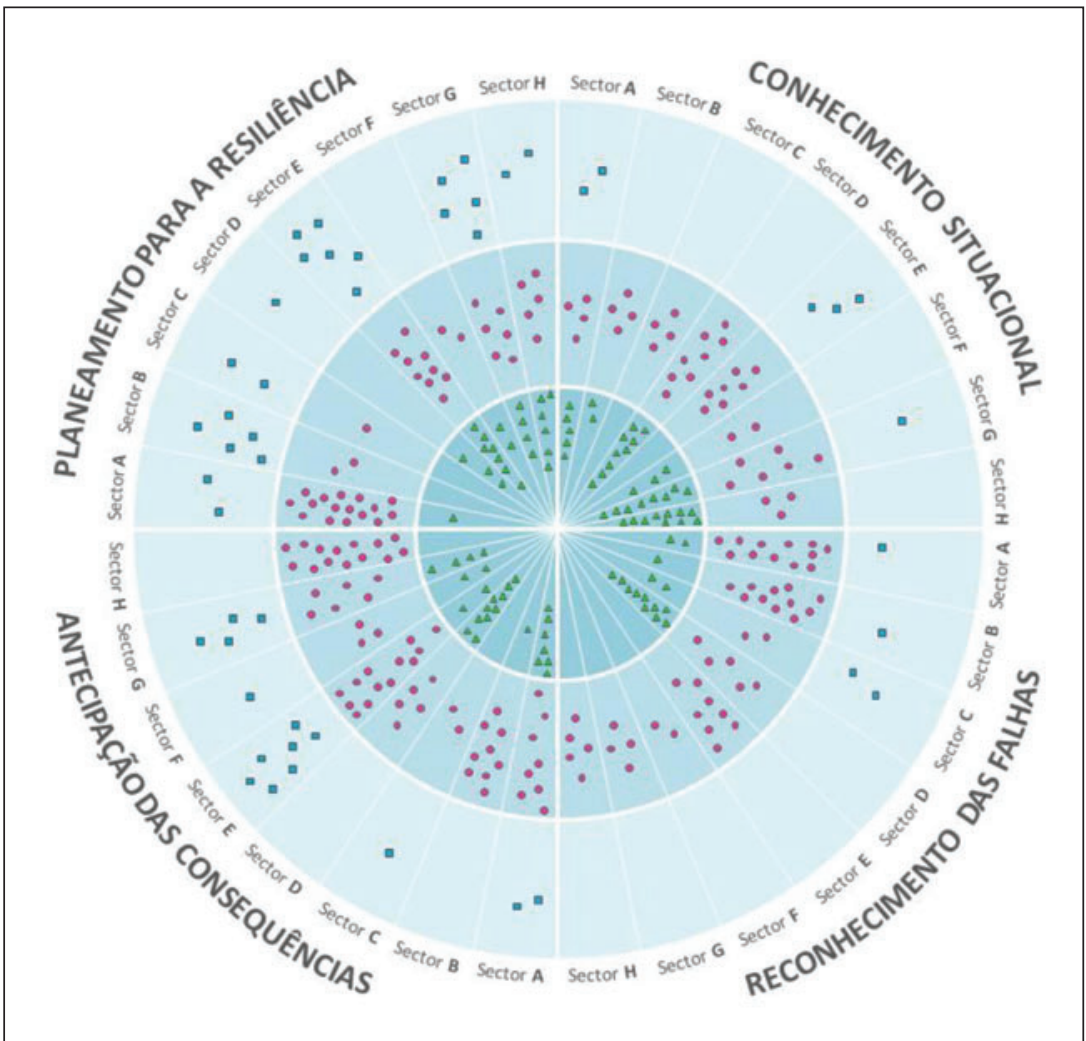

Gráfico 3 Comparação dos ecos das propriedades de protorresiliência dos setores na dimensâo do conhecimento

Dimensão:Conhecimento

Propriedades: Equilibrio,Optimzação e Flexibilidade

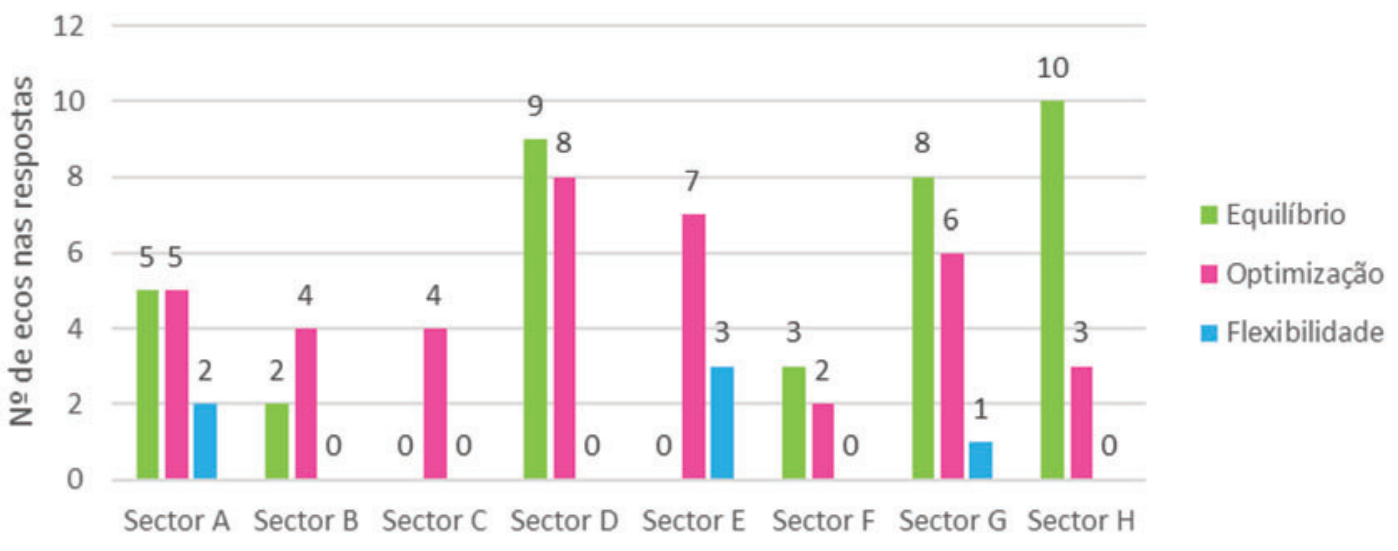

G (8 ecos). Comparativamente, na propriedade de otimização, os valores são mais lineares. Na propriedade de flexibilidade, os valores apresentam-se em geral baixos, com valores entre 3 (setor E) e 0 ecos (setores B, C, D, F e H). 
$61 \mid$ Revista de GESTÃo dos Países de Língua Portuguesa

\section{Dimensão do reconhecimento}

O Gráfico 4 permite identificar que, na dimensão do reconhecimento de falhas, a propriedade que se destaca mais é a otimização, apresentando valores mais elevados no setor A (12 ecos) e no setor B (11 ecos) por comparação com as restantes propriedades. As propriedades do equilíbrio e da flexibilidade apresentam valores incomparavelmente mais baixos, nomeadamente, entre 2 e 0 ecos, à exceção do equilíbrio, no setor D (10 ecos).

\section{Dimensão da antecipação}

O Gráfico 5 permite identificar que, na dimensão da antecipação de consequências, a propriedade que mais se destaca é a otimização, com valores

Gráfico 4 Comparação dos ecos das propriedades de protorresiliência dos setores na dimensâo do reconhecimento de falhas

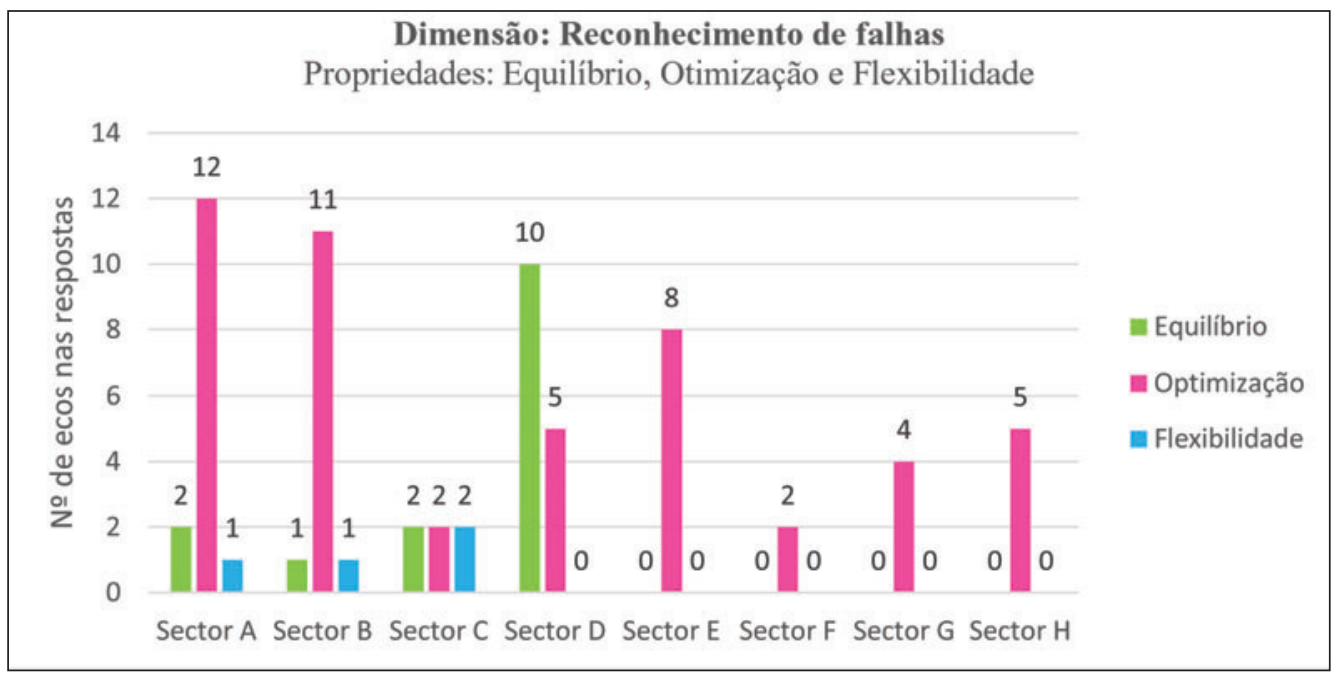

Gráfico 5 Comparação dos ecos das propriedades de protorresiliência dos setores na dimensâo da antecipaçâo

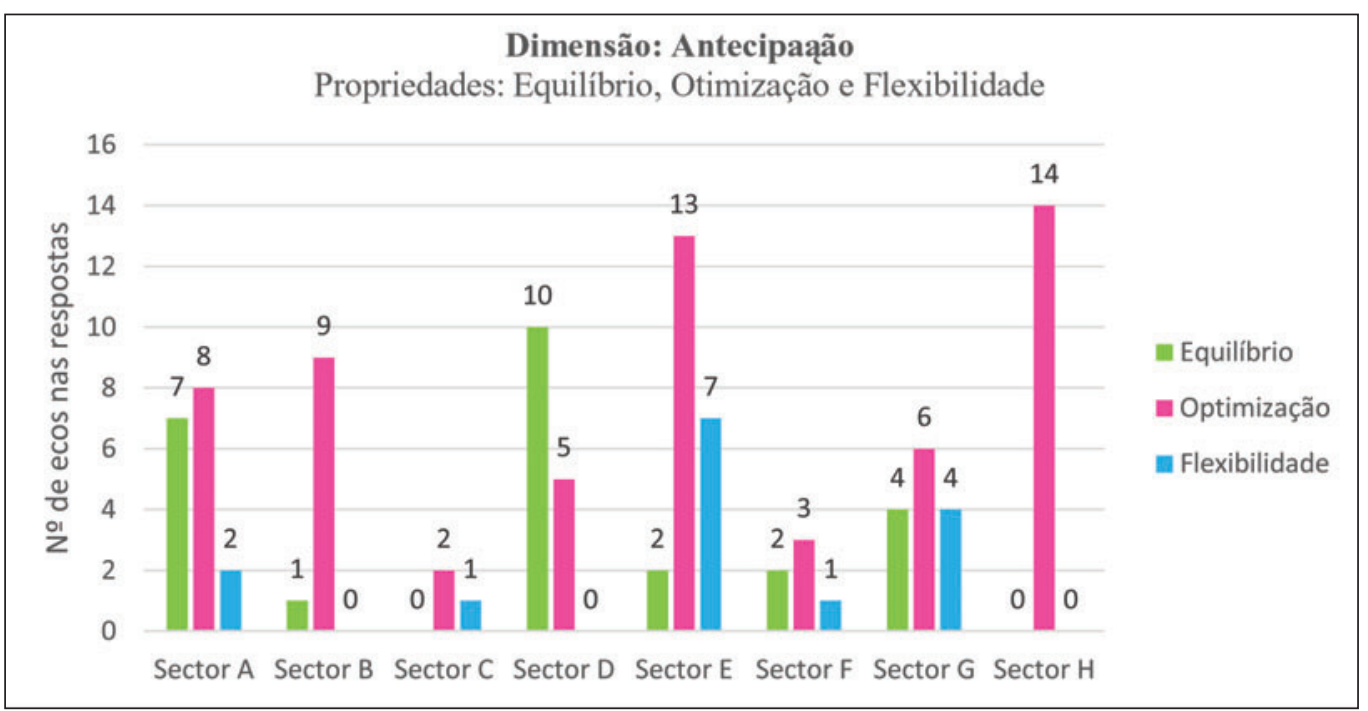


mais elevados no setor $\mathrm{H}$ (14 ecos) e no setor E (13 ecos). Relativamente à propriedade do equilíbrio, à exceção dos setores $\mathrm{D}$ (10 ecos) e A (7 ecos), os valores apresentam-se em geral baixos. Em relação à propriedade da flexibilidade, à exceção dos setores $\mathrm{E}$ ( 7 ecos) e $\mathrm{G}$ (4 ecos), os valores apresentam-se muito baixos, nomeadamente nos setores $\mathrm{B}, \mathrm{D}$ e $\mathrm{H}$ que apresentam 0 ecos. Note-se que, apesar do setor $\mathrm{H}$ apresentar 14 ecos de otimização, sendo o valor mais elevado do gráfico, apresenta 0 ecos tanto na propriedade do equilíbrio como na propriedade da flexibilidade.

\section{Dimensão de planeamento}

O Gráfico 6 permite identificar que, na dimensão do planeamento para a resiliência, à exceção dos ecos de Otimização nos setores A (16 ecos) e E (9 ecos), existem, em geral, valores relativamente baixos em todos os setores, quando comparados às dimensões anteriormente analisadas (conhecimento, reconhecimento de falhas e antecipação das consequências). Destacam-se as principais lacunas: no equilíbrio, os setores $\mathrm{B}$ e $\mathrm{C}$, com 0 ecos; na otimização, o setor D, com 0 ecos; e, na flexibilidade, o setor F, com 0 ecos. Sublinha-se que, na dimensão do planeamento, a flexibilidade apresenta, em geral, valores mais elevados em relação às restantes dimensões analisadas.

\section{Conclusões}

Construir a resiliência de uma organização, respeitando a sua cultura organizacional, pressupõe indagar a sua resiliência potencial; isto é, os recursos que estão disponíveis para produzir uma estrutura mais otimizada, de modo a superar as inúmeras adversidades que se levantam num mundo altamente competitivo e em processo de mudança.

Tendo em conta a literatura conhecida, este trabalho oferece um conjunto de contributos que concorrem para proporcionar aos decisores respostas

Gráfico 6 Comparação dos ecos das propriedades de protorresiliência dos setores na dimensâo do planeamento

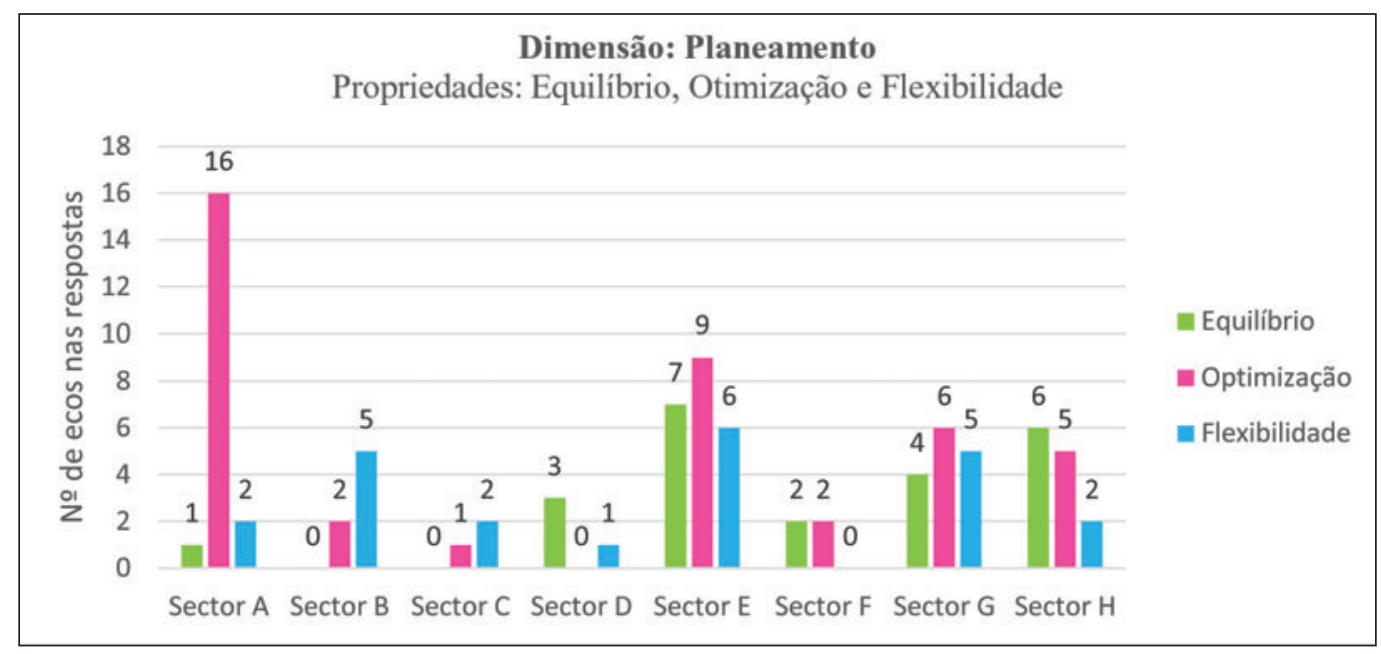


mais eficientes e eficazes. Um dos contributos deste trabalho está relacionado com o facto de que, pela primeira vez, é apontada uma forma simples de mapear com coordenadas (letras e números) do território concetual de uma organização, avaliando, simultaneamente, as dimensões e as propriedades da resiliência.

O mapeamento oferece um conjunto de potencialidades associadas que ainda estão em análise. Todavia um dos aspetos mais relevantes que foi identificado prende-se com a simplicidade e a eficiência do Radar-R. Com efeito, o mapeamento permite tomar consciência e avaliar todos os quadrantes do mapa. Através de uma única imagem sintetizadora é possível visualizar os pontos fortes, os pontos fracos, as fragilidades, as lacunas, o grau de alinhamento dos setores e o grau de consciência sobre os recursos, por forma a levar os setores a reconhecerem as potencialidades, necessidades, prioridades, etc.

Numa lógica de facilitar a todas as organizações uma possibilidade de conhecer a sua resiliência potencial, o Radar-R apresenta-se como uma ferramenta de fácil utilização e proliferação, o que significa que se poderá tornar uma ferramenta acessível e útil para gerar mudanças positivas na sociedade, de modo a permitir às organizações quer sociais, políticas ou económicas a superação de fragilidades, em tempos de incerteza.

Tendo em conta a relevância da temática e a escassez de publicações sobre resiliência em língua portuguesa, este artigo pretende contribuir para o desenvolvimento do estudo teórico e empírico da resiliência das organizações nas comunidades científicas e organizacionais/empresariais de língua portuguesa, no sentido de estimular a apropriação do conceito e o desenvolvimento de boas práticas.

\section{Referências bibliográficas}

CAVELTY, M. e PRIOR, T. (2013), «La résilience dans la politique de sécurité: Présent et Avenir». Politique de Sécurité, no 142. http://www.css.ethz.ch/publications/pdfs/CSS-Analysen-142-FR.pdf.

HOLLING, C. S. (1973), «Resilience and stability of ecological systems». Annual Review of Ecology and Systematics, 4, pp. 1-23.

HOLLING, C.S. (2001), "Understanding the complexity of economic, ecological, and social systems». Ecosystems, 4, pp. 390-405.

HOLLNAGEL, E. (2006), Resilience Engineering: Concepts and Precepts. Ashgate, Aldershot, UK.

MARINHA PORTUGUESA (2006), «Diretiva para as operações da Marinha. Paradigma operacional. O conceito do duplo uso». Diretiva 002/2006 do CEMA.PAA32 - Parte VIII. Documentação Estruturante da Estratégia Naval. Marinha, Lisboa.

MCMANUS, S.; SEVILLE, E.; VARGO, J. e BRUNSDON, D. (2008), «Facilitated process for improving organization resilience». Natural Hazards Review, 9(2), pp. 81-90.

PETIT, F.; BUEHRING, W.A.; WHITFIELD, R.; FISHER, R.E. e COLLINS, M.J. (2011), «Protective measures and vulnerability indices for enhanced critical infrastructure protection programme». International Journal of Critical Infrastructures, 7(3), pp. 1-20.

RIBEIRO, A.S. (2008), «Elaboração da estratégia de defesa militar: Contributo para um novo modelo». Tese de Doutoramento em Estratégia, Instituto Superior de Ciências Sociais e Políticas, Lisboa.
Através de uma única imagem sintetizadora é possível visualizar os pontos fortes, os pontos fracos, as fragilidades, as lacunas, o grau de alinhamento dos setores e o grau de consciência sobre os recursos, por forma a levar os setores a reconhecerem as potencialidades, necessidades, prioridades, etc. 
64 | Revista de GESTÃO dos Países de Língua Portuguesa

RIBEIRO, A.S.; SILVA, F.B.; PALMA, J.N. e MONTEIRO, N.S. (2010), «Estratégia naval portuguesa». Cadernos Navais, Grupo de Estudos e Reflexão Estratégica, n. ${ }^{\circ}$ 34. Edições Culturais da Marinha, Lisboa.

ROBERT, B. (2009), Organizacional Resilience - Concept and Evaluation Methods. Presses Internationales Polytechnique, Montréal, Canada.

RUTTER, M. (1993), «Resilience: Some conceptual considerations». Journal of Adolescent Health, 14(8), pp. 626-633.

THERRIEN, M.-C. (2010), «Stratégies de résilience et infrastructures essentielles». Téléscope, 16(2), pp. 154-171.

TIMOSHENKO, S. (1983), History of Strength of Materials. ON General Publishing Company, Toronto.

oUNITED NATIONS/OFFICE FOR DISASTER RISK REDUCTION (2015), «Sendai framework for disaster risk reduction 2015-2030». United Nations Office for Disaster Risk Reduction, Geneva.

WERNER, E.E. (1993), «Risk, resilience and recovery: Perspectives from the Kauai longitudinal study». Development and Psychopathology, 5, pp. 503-515. 